\title{
A Study of a Nonlinear Ordinary Differential Equation in Modular Function Spaces Endowed with a Graph
}

\author{
Jaauad Jeddi $\mathbb{D},{ }^{1}$ Mustapha Kabil, ${ }^{1}$ and Samih Lazaiz $\mathbb{D}^{2}$ \\ ${ }^{1}$ Laboratory of Mathematics and Applications, Faculty of Sciences and Technologies Mohammedia, \\ University Hassan II Casablanca, Morocco \\ ${ }^{2}$ LASMA Laboratory, Faculty of Sciences Dhar El Mahraz, University Sidi Mohamed Ben Abdellah, Fes, Morocco
}

Correspondence should be addressed to Jaauad Jeddi; jaauadjeddi@gmail.com

Received 4 November 2020; Revised 18 December 2020; Accepted 8 January 2021; Published 30 January 2021

Academic Editor: Huseyin Isik

Copyright (C) 2021 Jaauad Jeddi et al. This is an open access article distributed under the Creative Commons Attribution License, which permits unrestricted use, distribution, and reproduction in any medium, provided the original work is properly cited.

In this paper, we prove by means of a fixed-point theorem an existence result of the Cauchy problem associated to an ordinary differential equation in modular function spaces endowed with a reflexive convex digraph.

\section{Introduction}

It is well known that fixed-point theory is a powerful tool that was frequently exploited to prove existence of solutions of differential equations not only in Banach spaces but also in a wider range of spaces, particularly in Orlicz and Musielak-Orlicz spaces $[1,2]$ and more generally in modular function spaces.

The Orlicz spaces were introduced in the early 1930s when the lack of flexibility of classical Lebesgue function spaces $L^{p}$, in fact the lack of stability under some differential operators, leads Orlicz and Birnbaum to consider the space

$$
L \varphi=\left\{f: \mathbb{R} \longrightarrow \mathbb{R}: \int_{\mathbb{R}} \varphi(\lambda|f(x)|) d x \longrightarrow 0 \text { as } \lambda \longrightarrow 0\right\}
$$

where $\varphi: \mathbb{R}_{+} \mathbb{R}_{+}$is a convex increasing function such that $\lim _{x \rightarrow \infty} \varphi(x)=\infty$ (the convexity of $\varphi$ was subsequently very often omitted).

Later, in the end of the 1950s, Orlicz and Musielak considered the space

$$
L \phi=\left\{f \in X: \int_{\Omega} \phi(x, \lambda|f(x)|) d \mu \longrightarrow 0 \text { as } \lambda \longrightarrow 0\right\},
$$

where $\left(\Omega, \sum, \mu\right)$ is a measure space, $X$ is the set of all realvalued (or complex-valued) $\sum$-measurable, $\mu$-almost everywhere finite functions on $\Omega$, and $\phi: \Omega \times \mathbb{R}_{+} \longrightarrow \mathbb{R}_{+}$is a Caratheodory function which means that it is $\sum$-measurable for first variable, nondecreasing continuous mapping on the second variable and such that $\phi(x, 0)=0, \phi(x, u)>0$ if $u>0$.

The theory of modular function spaces was initiated by Kozlowski [3-5], and those spaces were a sort of spaces situated in between the Musielak-Orlicz and modular ones that were both more concrete of ordinary modular spaces, as treating about functions sets, and offering much more flexibility than the Musielak-Orlicz spaces.

Furthermore, in $[6,7]$ jointly with Khamsi, Kozlowski has initiated fixed-point results in modular function spaces. Recently, a new direction has been developed, combining fixed-point results and graph theory; see, for instance, [8-10].

In the same vein, Kozlowski in [11] managed to prove the existence of solutions of the following differential equation of type:

$$
\text { (O.D.E) }\left\{\begin{array}{l}
u(0)=f \\
u^{\prime}(t)+(I-T) u(t)=0, \forall t \in[0, A]
\end{array}\right.
$$

where $u(t)$ has values in modular function spaces and $T$ satisfies nonexpansiveness assumption. 
In this work, we intend to solve the equation (O.D.E) in modular function spaces endowed with a digraph, where nonexpansiveness of $T$ is restricted to connected points, which is a far more general result than the one obtained by Kozlowski. We will first establish a fixed-point result that will be employed to prove the existence of solutions of (O.D.E) with less restraint conditions over $T$.

\section{Preliminaries}

We begin by recalling some elementary notions about graphs; see [12] for further properties.

Definition 1. A directed graph or digraph $G$ is determined by a nonempty set $V(G)$ of its vertices and the set $E(G) \subset V(G$ )$\times V(G)$ of its directed edges. A digraph is reflexive if each vertex has a loop. Given a digraph $G=(V, E)$.

(i) If whenever $(x, y) \in E(G) \Rightarrow(y, x) \notin E(G)$, then the digraph $G$ is called an oriented graph

(ii) A digraph $G$ is transitive whenever $[(x, y) \in E(G)$ and $(y, z) \in E(G)] \Rightarrow(x, z) \in E(G)$, for any $x, y, z \in$ $V(G)$

(iii) A dipath of $G$ is a sequence $a_{0}, a_{1}, \cdots, a_{n}, \cdots$ with ( $\left.a_{i}, a_{i}+1\right) \in E(G)$ for each $i \in \mathbb{N}$

(iv) A finite dipath of length $\mathrm{n}$ from $x$ to $y$ is a sequence of $n+1$ vertices $\left(a_{0}, a_{1}, \cdots, a_{n}\right)$ with $\left(a_{i}, a_{i+1}\right) \in E(G)$ and $x=a_{0}, y=a_{n}$

(v) A closed directed path of length $n>1$ from $x$ to $y$, i.e., $x=y$, is called a directed cycle

(vi) A digraph is connected if there is a finite (di)path joining any two of its vertices and it is weakly connected if $G^{\sim}$ is connected

(vii) $[x]_{G}$ is the set of all vertices which are contained in some path beginning at $x$ (i.e., $y \in[x]_{G} \Leftrightarrow$ there exist $\left(a_{0}, a_{1}, \cdots, a_{n}\right)$ with $\left(a_{i}, a_{i+1}\right) \in E(G)$ and $x=a_{0}, y$ $\left.=a_{n}\right)$

We also need to introduce some properties of modular function spaces and tools that will be often used later. For more details, one can consult $[3-5,13,14]$.

Let $\Omega$ be a nonempty set and $\mathcal{P}$ a nontrivial $\delta$-ring of subsets of $\Omega$ and let $\Sigma$ be the smallest $\sigma$-algebra of subsets of $\Omega$ such that $\Sigma$ contains $\mathcal{P}$ such that $E \cap A \in \mathcal{P}$ for every $E \in \mathcal{P}$ and $A \in \sum$; and $K_{n} \uparrow \Omega$ where $K_{n} \in \mathcal{P}$, for all $n$.

$\mathcal{E}$ is the linear space of $\mathcal{P}$-simple functions, and $M_{\infty}$ is the set of measurable functions. We denote by $1_{A}$ the characteristic function of $A$, where $A \subset \Omega$.

Definition 2 [11]. An even convex function $\rho: M_{\infty} \longrightarrow[0,+$ $\infty]$ is called regular convex function pseudomodular if

(i) $\rho(0)=0$ (ii) $\rho$ is monotone, i.e., if for $f, g \in M_{\infty}, f(\omega) \leq g(\omega)$ for all $\omega \in \Omega$, then $\rho(f) \leq \rho(g)$

(iii) $\rho$ is orthogonally subadditive, i.e., $\rho\left(f .1_{A \cup B}\right)=\rho(f$ $\left..1_{A}\right)+\rho\left(f .1_{B}\right)$ whenever $A, B \in \sum$ and $A \cap B=\phi$ and $f \in \sum$

(iv) $\rho$ has the Fatou property, i.e., if $\left(\left|f_{n}(\omega)\right|\right)_{n} \uparrow|f(\omega)|$, for all $\omega \in \Omega, f_{n}, f \in M_{\infty}$ then $\rho\left(f_{n}\right) \uparrow \rho(f)$

(v) $\rho$ is order continuous in $\mathcal{E}$, i.e., $g_{n} \in \mathcal{E}$, and $\left|g_{n}\right| \downarrow 0$ implies $\rho\left|g_{n}\right| \downarrow 0$

Let $\rho$ be a regular convex function pseudomodular, the following notions are borrowed from [11].

(i) A set $A \in \sum$ is said to be $\rho$-null if $\rho\left(g .1_{A}\right)=0, \forall g \in \mathcal{E}$

(ii) A property $(P)$ is said to hold $\rho$ almost everywhere if the exceptional set is $\rho$-null

(iii) We will identify pair of measurable sets whose symmetric difference is $\rho$-null, as well as pair of measurable function differing only on a $\rho$-null set

(iv) $M\left(\Omega, \sum, \mathcal{P}, \rho\right)=\left\{f \in M_{\infty}:|f(\omega)|<\infty \rho-\right.$ a.e. $\}$ briefly noted $M$

(v) $\rho$ is said to be a regular convex function modular if $\rho(f)=0$ implies $f=0 \rho-$ a.e

(vi) We denote by $\mathfrak{R}$ the set of all nonzero regular convex function modulars on $\Omega$

Definition 3 [11]. Let $\rho \in \mathfrak{R}$.

(a) We say that $\left(f_{n}\right)_{n} \in L_{\rho} \rho$ converges to $f$ and write $f_{n}$ $\longrightarrow f(\rho)$, if $\rho\left(f_{n}-f\right) \longrightarrow 0$, and a sequence $\left(f_{n}\right)_{n}$ $\in L_{\rho}$ is called $\rho$-Cauchy if $\rho\left(f_{n}-f_{m}\right) \longrightarrow 0$ as $(n, m$ )$\longrightarrow \infty$

(b) A set $B \subset L_{\rho}$ is called $\rho$-closed, if for any sequence $\left(f_{n}\right)_{n} \in B, f_{n} \longrightarrow f(\rho)$ implies $f \in B$

(c) A set $B \subset L_{\rho}$ is called $\rho$-bounded, if its diameter $\delta_{\rho}($ $B)=\sup \{\rho(f-g): f, g \in B\}$ is finite

(d) A set $B \subset L_{\rho}$ is called $\rho$-compact, if for any sequence $\left(f_{n}\right)_{n} \in B$ there exists a subsequence $\left(f_{k_{n}}\right)_{n}$ and $f \in$ $B$ such that $f_{k_{n}} \rho$-converges to $f$

(e) A set $B \subset L_{\rho}$ is called $\rho$-a.e.-closed, if for any sequence $\left(f_{n}\right)_{n} \in B, f_{n} \longrightarrow f, \rho$-a.e. implies $f \in B$

(f) A set $B \subset L_{\rho}$ is called $\rho$-a.e.compact, if for any sequence $\left(f_{n}\right)_{n} \in B$ there exists a subsequence $\left(f_{n_{k}}\right)_{k}$ and $f \in B$ such that $f_{n_{k}} \longrightarrow f$, $\rho$-a.e

Definition 4. [11]. Let $\rho \in \mathfrak{R}$. 
The modular function space is the vector space $L_{\rho}\left(\Omega, \sum\right)$ or briefly $L_{\rho}$ is defined

$$
L_{\rho}=\left\{f \in \mathcal{M}: \lim _{\lambda \rightarrow 0} \rho(\lambda . f)=0\right\} .
$$

The map $\|\cdot\|_{\rho}: L_{\rho} \longrightarrow[0,+\infty)$ defined by

$$
\|f\|_{\rho}=\inf \left\{\alpha>0: \rho\left(\frac{f}{\alpha}\right) \leq 1\right\}
$$

is called norm of Luxembourg on $L_{\rho}$.

The following properties play a prominent role in the study of modular function spaces.

Definition 5 [11]. Let $\rho \in \mathfrak{R}$.

We say that $\rho$ has the $\Delta_{2}$-property, if $\rho\left(2 f_{n}\right) \longrightarrow 0$ whenever $\rho\left(f_{n}\right) \longrightarrow 0,\left(\left(f_{n}\right)_{n} \in L_{\rho}\right)$. We say that $\rho$ has the $\Delta_{2}$-type condition, if there exists $k \in[0,+\infty)$ such that $\rho(2 f) \leq k \rho(f)$, for any $f \in L_{\rho}$. [6].

The following definitions and results could be found in

Theorem 6. Let $\rho \in \Re$.

(i) $\left(L_{\rho},\|.\|_{\rho}\right)$ is a complete normed space, and $L \rho$ is $\rho$ -complete

(ii) $\left\|f_{n}\right\|_{\rho} \longrightarrow 0$ iff $\rho\left(\alpha f_{n}\right) \longrightarrow 0$ for every $\alpha>0$

(iii) If $\rho\left(f_{n}-f\right) \longrightarrow 0$ there exists $\left(f_{n_{k}}\right)_{k}$ subsequence of $\left(f_{n}\right)_{n}$ such that $f_{n_{k}} \longrightarrow f, \rho$-a.e

(iv) If $f_{n} \longrightarrow f \rho-a . e$, then $\rho(f) \leq \liminf _{n \rightarrow+\infty} \rho\left(f_{n}\right)$ (the Fatou property)

(v) If $\rho$ has the $\Delta_{2}$-property and $\rho\left(\alpha f_{n}\right) \longrightarrow 0$ for $\alpha>0$, then $\left\|f_{n}\right\|_{\rho} \longrightarrow 0$

Definition 7. Let $\rho \in \Re$, we define

$$
\begin{gathered}
L_{\rho}^{o}=\left\{f \in L_{\rho}: \rho(f, .) \text { is order continuous }\right\}, \\
E_{\rho}=\left\{f \in L_{\rho}: \lambda f \in L_{\rho}^{0}, \forall \lambda>0\right\} .
\end{gathered}
$$

Theorem 8. Let $\rho \in \mathfrak{R}$, then $E_{\rho}$ is a \|\|$_{\rho}$-closed subspace of $L_{\rho}$

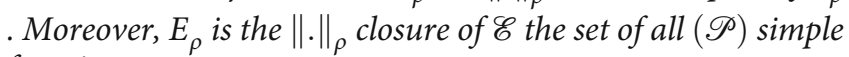
functions.

Definition 9. We say that a set $C \subset L_{\rho}$ possesses the Vitali property if $C \subset E_{\rho}$, and for any $g \subset L_{\rho}$ and $\left(g_{n}\right)_{n} \subset C$ with $g_{n} \longrightarrow g(\rho)$, there exists a subsequence $\left(g_{n_{k}}\right)_{k}$ of $\left(g_{n}\right)_{n}$ such that for every $\alpha>0$ the subadditive measures $\rho\left(\alpha g_{n_{k}}\right.$, .) are order equicontinuous. That is, if $\left(E_{p}\right)_{p} \subset \sum$ such that $\left(E_{p}\right)_{p} \downarrow$ $\varnothing$ then $\forall \alpha>0 \lim _{\rho \rightarrow \infty} \sup _{k \varepsilon N} \rho\left(\alpha g_{n k}, E_{p}\right) 0$.

The following statement characterizes sets with the Vitali property as subsets of $E_{\rho}$ where the $\rho$ convergence is equivalent to the $\|\bullet\|_{\rho}$ convergence.

Theorem 10. Let $\rho \in \mathfrak{R}$. A set $C \in L_{\rho}$ has the Vitali property if and only if the following conditions are satisfied:

(i) $C \subset E_{\rho}$

(ii) If $g \in L_{\rho}$ and $\left(g_{n}\right)_{n} \subset C$ and $\left(g_{n}\right)_{n} \longrightarrow g(\rho)$, then $\left\|g_{n}-g\right\|_{\rho} \longrightarrow 0$

Definition 11. A convex function modular $\rho \in \mathfrak{R}$ is said separable if $\forall f \in \mathcal{E},\left(\left\|f 1_{(.)}\right\|_{\rho}\right)$ is a separable set function for each $f \in \mathcal{E}$, which means that there exists a countable $\mathscr{A} \subset \mathscr{P}$ such that to every $A \in \mathscr{P}$ there corresponds a sequence $\left(A_{k}\right)_{k}$ of elements of $A$ with

$$
\forall \alpha>0, \rho\left(\alpha f, A \Delta A_{k}\right) \underset{k \rightarrow \infty}{\longrightarrow} 0 .
$$

We recall this important result, which states that if $\rho$ is separable, then $\left(L_{\rho},\|\cdot\|_{\rho}\right)$ is a separable Banach space; it is then a Polish space.

Theorem 12. Let $\rho \in \mathfrak{R}$. The space $\left(L_{\rho},\|\cdot\|_{\rho}\right)$ is separable if and only if $\rho$ is separable.

Remark 13. Let $Z$ be a separable linear subspace of $E_{\rho},\|\cdot\|_{\rho}$ and let $C \subset Z$ have the Vitali property. Assume that the function $u:[a, b] \longrightarrow C(a, b \in \mathbb{R})$, is $\rho$-continuous. Then, $u$ is Bochner integrable function with respect to the Lebesgue measure $m$ on $[a, b]$. That is, if for $\tau:=t_{0}<t_{1}<\cdots<t_{m}$ a subdivision of $[a, b]$, we define $|\tau|=\sup _{0 \leq i \leq m-1}\left(t_{i+1}-t_{i}\right)$ (called the step of $\tau$ ), then

$$
\lim _{|\tau| \rightarrow 0} \sum_{i=0}^{m-1}\left(t_{i+1}-t_{i}\right) u\left(t_{i}\right) \text { exists, }
$$

and we write

$$
\int_{a}^{b} u(s) d s=\lim _{|\tau| \rightarrow 0} \sum_{i=0}^{m-1}\left(t_{i+1}-t_{i}\right) u\left(t_{i}\right) .
$$

\section{Main Results}

Definition 14. Let $C \subset L_{\rho}, a, b, \in \mathbb{R}$ and $C \subset L_{\rho}$.

(i) A function $u:[a, b] \longrightarrow C$ is said to be continuous if $u\left(t_{n}\right) \longrightarrow u(t)(\rho)$ provided $t_{n} \longrightarrow t$. We denote by $\mathcal{C}([a . b], C)$ the set of all these continuous functions 
(ii) A mapping $T: C \longrightarrow C$ is said to be $\rho$-continuous if $T\left(f_{n}\right) \longrightarrow T(f)(\rho)$ provided $f_{n} \longrightarrow f(\rho)$

Proposition 15. Let $\rho \in \Re$ be separable and $C \subset E_{\rho}$ be a nonempty convex and $\rho$-closed set that has the Vitali property. Let $T: C \longrightarrow C$ be a $\rho$-continuous mapping and $f \in C$. Then, for every $u \in \mathcal{C}([0, A], C)$ the mapping $\phi(u)$ defined by

$$
\phi(u)(t)=e^{-t} f+\int_{0}^{t} e^{s-t} T(u(s)) d s \text { for every } t \in[0, A]
$$

takes values in $C$ and is continuous, i.e., $\phi(u) \in \mathcal{C}([0, A], C)$.

Proof. Let $u \in C([0, A], C)$. We will first prove that $\phi(u)(t)$ $\in C$ for every $t \in(0, A)$. Let $t \in(0, A)$, for $\tau:=t_{0}<t_{1}<\cdots<$ $t_{m}$, a subdivision of $[0, t]$, we define

$$
S_{\tau}(T(u))(t)=\sum_{i=0}^{m-1}\left(t_{i+1}-t_{i}\right) e^{t_{i}-t} T(u(t)) .
$$

By definition of Bochner integral

$$
\int_{0}^{t} e^{s-t} T(u(s)) d s=\lim _{|\tau| \rightarrow 0} S_{\tau}(T(u))(t) .
$$

We have $f \in C, T\left(u\left(t_{i}\right)\right) \in C$ for every $i \in\{1, \cdots, n\}$ and

$$
e^{-t}+\sum_{i=0}^{m-1}\left(t_{i+1}-t_{i}\right) e^{t_{i}-t} \leq e^{-t}+\int_{0}^{t} e^{s-t} d s=1
$$

Using convexity of $C$, we get

$e^{-t} f+\sum_{i=0}^{m-1}\left(t_{i+1}-t_{i}\right) e^{t_{i}-t} T\left(u\left(t_{i}\right)\right)+\left(1-\left(e^{-t}+\sum_{i=0}^{m-1}\left(t_{i+1}-t_{i}\right) e^{t_{i}-t}\right)\right) f \in C$. Thus,

Since $C$ is $\rho$-closed, it is also closed with respect to $\|\cdot\|_{\rho}$.

$$
\lim _{|\tau| \rightarrow 0}\left[e^{-t} f+\sum_{i=0}^{m-1}\left(t_{i+1}-t_{i}\right) e^{t_{i}-t} T\left(u\left(t_{i}\right)\right)+\left(1-\left(e^{-t}+\sum_{i=0}^{m-1}\left(t_{i+1}-t_{i}\right) e^{t_{i}-t}\right)\right) f \in C\right] .
$$

Observing that

$$
\lim _{|\tau| \rightarrow 0}\left(1-\left(e^{-t}+\sum_{i=0}^{m-1}\left(t_{i+1}-t_{i}\right) e^{t_{i}-t}\right)\right)=1-e^{-t}-\int_{0}^{t} e^{s-t} d s=0,
$$

we conclude

$$
\phi(u)(t)=e^{-t} f+\int_{0}^{t} e^{s-t} T(u(s)) d s \in C .
$$

Furthermore, as $C$ has the Vitali property, $T$ is continuous with respect to the norm of Luxembourg, and then $\phi(u$ ) is continuous as $t \mapsto \int_{0}^{t} e^{s-t} T(u(s)) d s$ is continuous; it is even differentiable.

The following notion of convex digraph was already introduced in [15].

Definition 16. Let $C \in L_{\rho}$ be a convex subset and $G$ a digraph $(E(g)=C)$, we say that $G$ is convex if $\forall \lambda \in[0,1]$ and $\forall f, g$, $u, v, \in C$ such that $f \in[u]_{G}$ and $g \in[v]_{G}$; then

$$
\lambda f+(1-\lambda) g \in[\lambda u+(1-\lambda) v]_{G} .
$$

Definition 17. Let $\rho \in \Re$ and $G$ a digraph $E(G) \subset L_{\rho}$. We say that $G$ has the $\left(P_{0}\right)$ property, if for all $\left(f_{n}\right)_{n},\left(g_{n}\right)_{n}, f, g \in L_{\rho}$ ,$g \in[f]_{G}$ provided $g_{n} \in\left[f_{n}\right]_{G}$ for everyn $\in \mathbb{N}$ and $f_{n} \longrightarrow f($ $\rho), g_{n} \longrightarrow g(\rho)$.

Definition 18. Let $C \subset L_{\rho}, G$ a digraph $E(G)=C$ and $T: C$ $\longrightarrow C$. We say that $T$ is $G$-monotone $\rho$-nonexpansive if for all $f, g \in C$ such that $g \in[f]_{G}$ we have

$$
\begin{gathered}
T(g) \in[T(f)]_{G}, \\
\rho(T(f)-T(g)) \leq \rho(f-g) .
\end{gathered}
$$

The following lemma will play a preponderant role in the proof of the next theorem; its proof can be found in [11].

Lemma 19. Let $\rho \in \Re$ be separable. Let $x, y:[0, A] \longrightarrow L_{p}$ two Bochner integrable $\|\cdot\|_{\bar{\rho}}$ bounded functions, where $A>0$. Then, for every $t \in[0, A]$, we have

$$
\rho\left(e^{-t} y(t)+\int_{0}^{t} e^{s-t} x(s) d s\right) \leq e^{-t} \rho(y(t))+\left(1-e^{-t}\right) \sup _{s \in[0, t]}(\rho(x(s))) .
$$

Now, we are able to state the main result.

Theorem 20. Let $\rho \in \Re$ be separable, $C \subset E_{\rho}$ a nonempty convex, $\rho$-bounded, $\rho$-closed set that has the Vitali property, and let $G(E(G)=C)$ be a reflexive, convex digraph, with property $\left(P_{0}\right)$. Let $T: C \longleftarrow C$ be a $\rho$-continuous and $G$-monotone $\rho$ -nonexpansive mapping and suppose there exists $f \in C$ such that $T(f) \in[f]_{G}$; then, the mapping

$$
\begin{gathered}
\phi: \mathcal{C}([0, A], C) \longrightarrow \mathcal{C}([0, A], C) \\
u \mapsto \phi(u)
\end{gathered}
$$

has a fixed point, where $\phi(u)(t)=e^{-t} f+\int_{0}^{t} e^{s-t} T(u(s)) d s$, for every $t \in[0, A]$. 
Proof. Note that the mapping $\varphi$ is well defined by Proposition 15 . We define the sequence $\left(u_{n}\right)_{n}$ by

$$
\begin{cases}u_{0}(t)=f, & \forall t \in[0, A] \\ u_{n+1}=\phi\left(u_{n}\right), & \forall n \in \mathbb{N}\end{cases}
$$

It is easy to see that $\left(u_{n}\right)_{n} \subset \mathcal{C}([0, A], C)$. We will prove by induction over $n \in \mathbb{N}$ that

$\forall t \in[0, A], \forall n, p \in \mathbb{N}, \rho\left(u_{n+p}(t)-u_{n}(t)\right) \leq\left(1-e^{A}\right)^{n+1} \delta_{\rho}(C)$.

For $n=0$, it comes for every $t$ in $[0, A]$,

$u_{p}(t)-u_{0}(t)=\int_{0}^{t} e^{s-t} T\left(u_{p-1}\right)(s) d s-\left(1-e^{-t}\right) f=\int_{0}^{t} e^{s-t}\left(T\left(u_{p-1}\right)(s)-f\right) d s$.

Lemma 19 applied for $y=0$ and $x(t)=T\left(u_{p-1}(t)\right)-f$ for every $t \in[0, A]$ gives

$\forall t \in[0, A], \rho\left(u_{p}(t)-u_{0}\right) \leq\left(1-e^{-t}\right) \sup _{s \in[0, t]} \rho\left(T\left(u_{p-1}(s)\right)-f\right) \leq\left(1-e^{-A}\right) \delta_{\rho}(C)$.

We suppose now that for all $t \in[0, A]$ and for all $p \in \mathbb{N}$

$$
\rho\left(u_{n+p}(t)-u_{n}(t)\right) \leq\left(1-e^{-A}\right)^{n+1} \delta_{\rho}(C)
$$

Let us first prove that $u_{n+1}(t) \in\left[u_{n}(t)\right]_{G}, \forall n \in \mathbb{N}$ and $\forall t$ $\in[0, A]$. By induction on $n$, for $n=0$, we have $\forall t \in[0, A]$,

$$
u_{1}(t)=e^{-t} f+\left(1-e^{-t}\right) T(f) \in\left[u_{0}(t)\right]_{G}=[f]_{G}
$$

as $G$ is convex and $T(f) \in[f]_{G}$. If we suppose that for every $t \in[0, A], u_{n+1}(t) \in\left[u_{n}(t)\right]_{G}$, then for $\tau:=t_{0}<t_{1} \cdots<t_{m}$ a subdivision of $[0, t]$. Set for $k \geq 1$

$$
u_{k}^{\tau}=e^{-t} f+\sum_{i=0}^{m-1}\left(t_{i+1}-t_{i}\right) e^{t_{i}-t} T\left(u_{k-1}\left(t_{i}\right)\right)
$$

then

$$
u_{n+2}^{\tau}=e^{-t} f+\sum_{i=0}^{m-1}\left(t_{i+1}-t_{i}\right) e^{t_{i}-t} T\left(u_{n+1}\left(t_{i}\right)\right)
$$

as $G$ is convex and $T\left(u_{n+1}\left(t_{i}\right)\right) \in\left[T\left(u_{n}\left(t_{i}\right)\right)\right]_{G}$ for every $i=1$ $, \cdots, m$ we have

$$
u_{n+2}^{\tau}+\left(1-\left(e^{-t}+\sum_{i=0}^{m=1}\left(t_{i+1}-t_{i}\right)^{t_{i}-t}\right)\right) f \in\left[u_{n+1}^{\tau}+\left(1-\left(e^{-t} \sum_{i=0}^{m-1}\left(t_{i+1}-t_{i}\right) e^{t_{i}-t}\right)\right) f\right]_{G}
$$

since $G$ has the $\left(P_{0}\right)$ property it follows that

$$
\lim _{|\tau| \rightarrow 0} u_{n+2}^{\tau}+\left(1-\left(e^{-t}+\sum_{i=0}^{m-1}\left(t_{i+1}-t_{i}\right) e^{t_{i}-t}\right)\right) f \in\left[\lim _{|\tau| \rightarrow 0} u_{n+1}^{\tau}+\left(1-\left(e^{-t}+\sum_{i=0}^{m-1}\left(t_{i+1}-t_{i}\right) e^{t_{i}-t}\right)\right) f\right]_{G},
$$

which is exactly $u_{n+2}(t) \in\left[u_{n+1}(t)\right]_{G}$. And then $u_{n+1}(t) \in$ $\left[u_{n}(t)\right]_{G}$ and $u_{n+p}(t) \in\left[u_{n}(t)\right]_{G}$ for every $n, p \in \mathbb{N}$ and $t \in[0$, A].

Now, as $\rho\left(u_{n+1+p}(t)-u_{n+1}(t)\right)=\rho\left(\int_{0}^{t} e^{s-t}\left(T\left(u_{n+p}(s)\right)-T\right.\right.$ $\left.\left.\left(u_{n}(s)\right)\right) d s\right)$, applying again Lemma 19 for $y(t)=0, x(t)=T$ $\left(u_{n+p}(s)\right)-T\left(u_{n}(s)\right)$, we get

$\rho\left(u_{n+1+p}(t)-u_{n+1}(t)\right) \leq\left(1-e^{-t}\right) \sup _{s \in[0, t]} \rho\left(T\left(u_{n+p}(s)\right)-T\left(u_{n}(s)\right)\right), \forall t \in[0, A]$,

but $\rho\left(T\left(u_{n+p}(s)\right)-T\left(u_{n}(s)\right)\right) \leq \rho\left(u_{n+p}(s)-u_{n}(s)\right), \forall s \in[0, t]$ (as $u_{n+p}(s) \in\left[u_{n}(s)\right]_{G}$ and $T$ is $G$-monotone $\rho$-nonexpansive); consequently,

$$
\rho\left(u_{n+1+p}(t)-u_{n+1}(t)\right) \leq\left(1-e^{t}\right) \sup _{s \in[0, t]} \rho\left(\left(u_{n+p}(s)\right)-\left(u_{n}(s)\right)\right), \forall t \in[0, A],
$$

by the inductive assumption, we get

$$
\rho\left(u_{n+1+p}(t)-u_{n+1}(t)\right) \leq\left(1-e^{-t}\right)\left(1-e^{-A}\right)^{n+1} \delta_{p}(C), \forall t \in[0, A],
$$

i.e.,

$$
\rho\left(u_{n+1+p}(t)-u_{n+1}(t)\right) \leq\left(1-e^{-A}\right)^{n+2} \delta_{\rho}(C), \forall t \in[0, A] .
$$

Using inequality (23), it is clear that for every $t \in[0, A]$, $\left(u_{n}(t)\right)_{n}$ is a $\rho$-Cauchy sequence in $C$. Since $C$ is $\rho$-closed in $L_{\rho}$, then it is $\rho$-complete and then $\left(u_{n}(t)\right)_{n} \rho-$ converges to some $u(t) \in C$, and thus, it converges to $u(t)$ with respect to $\|.\| \rho$. We also have $\forall t \in[0, A]$, and $\forall n \in \mathbb{N}, u(t) \in\left[u_{n}(t)\right]_{G}$ as $G$ has $\left(P_{0}\right)$ property. Indeed, for $n \in \mathbb{N}, u_{n+p}(t) \in\left[u_{n}(t)\right] G$ ,$\forall p \in \mathbb{N}$ and when $p \longrightarrow \infty, u(t) \in\left[u_{n}(t)\right]_{G}$.

Now, for $t \in[0, A]$, let $\tau:=t_{0}<t_{1}<\cdots<t_{m}$ be a subdivision of $[0, t]$. We have

$$
\begin{aligned}
& \rho\left(S_{\tau}\left(T\left(u_{n}(t)\right)\right)-S_{\tau}(T(u(t)))\right) \\
& \quad=\rho\left(\sum_{i=0}^{m-1}\left(t_{i+1}-t_{i}\right) e^{t_{i}-t}\left(T\left(u_{n}\left(t_{i}\right)\right)-T\left(u\left(t_{i}\right)\right)\right)\right),
\end{aligned}
$$

and by convexity of $\rho$

$$
\rho\left(S_{\tau}\left(T\left(u_{n}(t)\right)\right)-S_{\tau}(T(u(t)))\right) \leq \sum_{i=0}^{m-1}\left(t_{i+1}-t_{i}\right) e^{t_{i}-t} \rho\left(T\left(u_{n}\left(t_{i}\right)\right)-T\left(u\left(t_{i}\right)\right)\right) .
$$


Since $u\left(t_{i}\right) \in\left[u_{n}\left(t_{i}\right)\right]_{G}$ for every $i=0,1, \cdots, m$ and $T$ is $G$ -monotone $\rho$-nonexpansive

$\rho\left(S_{\tau}\left(T\left(u_{n}(t)\right)\right)-S_{\tau}(T(u(t)))\right) \leq \sum_{i=0}^{m-1}\left(t_{i+1}-t_{i}\right) e^{t_{i}-t} \rho\left(u_{n}\left(t_{i}\right)-u\left(t_{i}\right)\right) \underset{n \rightarrow \infty}{\longrightarrow} 0$,

that is, $\lim _{n \rightarrow \infty} \rho\left(S_{\tau}\left(T\left(u_{n}(t)\right)\right)-S_{\tau}(T(u(t)))\right)=0$.

Hence,

$$
\lim _{n \rightarrow \infty}\left\|S_{\tau}\left(T\left(u_{n}(t)\right)\right)-S_{\tau}(T(u(t)))\right\|_{\rho}=0
$$

Now, for every $t \in[0, A]$ and $n \in \mathbb{N}$

$$
\begin{aligned}
& \left\|S_{\tau}\left(T(u(t))-u(t)+e^{-f}\right)\right\|_{\rho} \leq\left\|S_{\tau}\left(T(u(t))-S_{\tau}\left(T\left(u_{n}(t)\right)\right)\right)\right\|_{\rho} \\
& \quad+\left\|S_{\tau}\left(T\left(u_{n}(t)\right)\right)-\int_{0}^{t} e^{s-t} T\left(u_{n}(s)\right) d s\right\|_{\rho} \\
& \quad+\left\|\int_{0}^{t} e^{s-t} T\left(u_{n}(s)\right) d s-u(t)+e^{-t} f\right\|_{\rho}
\end{aligned}
$$

From $\quad\left\|\int_{0}^{t} e^{s-t} T\left(u_{n}(s)\right) d s-u(t)+e^{-t} f\right\|_{\rho}=$ $\left\|u_{n+1}(t)-u(t)\right\|_{\rho}$, we get

$$
\lim _{|\tau| \rightarrow 0}\left\|S_{\tau}(T(u(t)))-u(t)+e^{-t} f\right\|_{\rho}=0
$$

i.e., $s \mapsto e^{(s-t)} T(u(s))$ is Bochner integrable and $\int_{0}^{t} e^{s-t} T(u(s)$ )$d s=u(t)-e^{-t} f$. Finally, we get for every $t \in[0, A]$

$$
\phi(u)(t)=e^{-t} f+\int_{0}^{t} e^{s-t} T(u(s)) d s=u(t),
$$

that is, $u$ is a fixed point of $\phi$.

A similar result can be obtained without assuming the Vitali property, but we need to assume that $\rho$ has the $\Delta_{2}$ -property.

Theorem 21. Let $\rho \in \Re$ be separable and has the $\Delta_{2}$-property, $C \subset L_{\rho}$ a nonempty convex, $\rho$-bounded, $\rho$-closed set, and let $G(E(G)=C)$ be a reflexive, convex digraph, with property ( $\left.P_{0}\right)$. Let $T: C \longrightarrow C$ be a $\rho$-continuous and G-monotone $\rho$ -nonexpansive mapping, and suppose that there exist $f \in C$ such that $T(f) \in[f]_{G}$; then, the mapping

$$
\begin{gathered}
\phi: \mathcal{C}([0, A], C) \longrightarrow \mathcal{C}([0, A], C) \\
u \mapsto \phi(u)
\end{gathered}
$$

has a fixed point where $\phi(u)(t)=e^{-t} f+\int_{0}^{t} e^{s-t} T(u(s)) d s$, for every $t \in[0, A]$.

Proof. Since $\rho$ has the $\Delta_{2}$-property, the $\rho$-convergence is equivalent to the convergence with respect to $\|.\|_{\rho}$ all over in $L_{\rho}$; the proof of this corollary runs along similar lines to the proof of Theorem 20 .

The last result is devoted to prove the existence of solution of the equation (O.D.E).

Theorem 22. Let $\rho \in \mathfrak{R}$ be separable, $C \subset E_{\rho}$ a nonempty convex, $\rho$-bounded, $\rho$-closed set that has the Vitali property, and let $G(E(G)=C)$ be a reflexive, convex digraph, with property $\left(P_{0}\right)$. Let $T: C \longrightarrow C$ be a $\rho$-continuous and $G$-monotone $\rho$ -nonexpansive mapping, and suppose that there exists $f \in C$ such that $T(f) \in[f]_{G}$; then, the differential equation

$$
(\text { O.D.E })\left\{\begin{array}{l}
u(0)=f, \\
u^{\prime}(t)+(I-T) u(t)=0, \forall t \in[0, A]
\end{array}\right.
$$

where $u:[0, A] \longrightarrow C, A>0$, has a solution.

Proof. The application $\phi$ defined above has a fixed point $u$ $\in \mathcal{C}([0, A], C)$, that is,

$$
u(t)=e^{-t} f+\int_{0}^{t} e^{s-t} T(u(s)) d s, \forall \in[0, A],
$$

then $u$ is differentiable and

$$
\begin{aligned}
u^{\prime}(t) & =-e^{-t} f-\int_{0}^{t} e^{s-t} T(u(s)) d s+e^{-t}\left(e^{t} T(u(t))\right) \\
& =T(u(t))-u(t), \forall t \in[0, A]
\end{aligned}
$$

that is, $u$ is the solution of (O.D.E).

Note that the result of Theorem 22 remains true if $\rho$ has the $\Delta_{2}$-property instead of $C$ having the Vitali property.

\section{Data Availability}

No data were used to support this study.

\section{Conflicts of Interest}

The authors declare that there are no conflicts of interest regarding the publication of this paper.

\section{References}

[1] A. Benkirane and M. Sidi El Vally, "An existence result for nonlinear elliptic equations in Musielak-Orlicz-Sobolev spaces," Bulletin of the Belgian Mathematical Society - Simon Stevin, vol. 20, no. 1, pp. 57-75, 2013.

[2] J. J. Nieto and R. Rodríguez-López, "Contractive mapping theorems in partially ordered sets and applications to ordinary differential equations," Order, vol. 22, no. 3, pp. 223-239, 2005.

[3] W. M. Kozlowski, Modular Function Spaces, Marcel Dekker, Inc., 1988.

[4] W. M. Kozlowski, "Notes on modular function spaces I," Commentationes Mathematicae, vol. 28, no. 1, pp. 91-104, 1988. 
[5] W. M. Kozlowski, "Notes on modular function spaces II," Commentationes Mathematicae, vol. 28, no. 1, pp. 105-120, 1988.

[6] M. A. Khamsi and W. M. Kozlowski, Fixed Point Theory in Modular Function Spaces, Birkhäuser, 2015.

[7] M. A. Khamsi, W. M. Kozlowski, and S. Reich, "Fixed point theory in modular function spaces," Nonlinear Analysis: Theory, Methods \& Applications, vol. 14, no. 11, pp. 935-953, 1990.

[8] M. R. Alfuraidan and M. A. Khamsi, "On Gregus-Ćirić mappings on weighted graphs," Fixed Point Theory, vol. 20, no. 1, pp. 19-30, 2019.

[9] J. Jeddi, M. Kabil, and S. Lazaiz, "Common fixed-point theorems in modular function spaces endowed with reflexive digraph," International Journal of Mathematics and Mathematical Sciences, vol. 2020, Article ID 9794134, 5 pages, 2020.

[10] A. Nicolae, D. O’Regan, and A. Petrusel, "Fixed point theorems for singlevalued and multivalued generalized contractions in metric spaces endowed with a graph," Georgian Mathematical Journal, vol. 18, no. 1, pp. 307-327, 2011.

[11] W. M. Kozlowski, "On nonlinear differential equations in generalized Musielak-Orlicz spaces," Commentationes Mathematicae, vol. 53, no. 2, pp. 13-33, 2013.

[12] M. Rigo, Advanced Graph Theory and Combinatorics, John Wiley \& Sons, 2016.

[13] T. Dominguez-Benavides, M. A. Khamsi, and S. Samadi, "Asymptotically regular mappings in modular function spaces," Scientiae Mathematicae Japonicae, vol. 53, no. 2, pp. 295-304, 2001.

[14] J. Musielak, Orlicz Spaces and Modular Spaces, Springer-Verlag, 1983.

[15] J. Jeddi, M. Kabil, and S. Lazaiz, "Some fixed point theorems in modular function spaces endowed with a graph," Abstract and Applied Analysis, vol. 2020, Article ID 2135859, 7 pages, 2020. 